\title{
Visualization strategies for major white matter tracts for intraoperative use
}

\author{
Christopher Nimsky ${ }^{1,2}$, Oliver Ganslandt ${ }^{1,2}$, Frank Enders ${ }^{2}$, Dorit Merhof ${ }^{2}$, Thilo \\ Hammen $^{2}$, Michael Buchfelder ${ }^{1,2}$ \\ ${ }^{1}$ Department of Neurosurgery, University Erlangen-Nuremberg \\ ${ }^{2}$ Neurocenter, University Erlangen-Nuremberg
}


Streamline representation of major fiber tract systems along with high-resolution anatomical data provides a reliable orientation for the neurosurgeon. For intraoperative visualization of these data either on navigation screens near the surgical field or directly in the surgical field applying headsup displays of operating microscopes, wrapping of all streamlines of interest to render an individual object representing the whole fiber bundle is the most suitable representation. Integration of fiber tract data into a neuronavigation setup allows removal of tumors adjacent to eloquent brain areas with low morbidity.

Keywords: diffusion tensor imaging, glyph representation, fiber tracking, functional neuronavigation, visualization strategies, major white matter tracts 


\section{Introduction}

In white matter of the brain, diffusion is anisotropic, i.e. the water diffusion is not equal in all three orthogonal directions. This is due to the strong aligned microstructure, including cell membranes and the myelin sheath surrounding myelineated white matter, causing impediment of the water motion. Isotropic diffusion can be graphically represented as a sphere, whereas anisotropic diffusion can be graphically expressed as an ellipsoid, with water molecules moving farther along the long axis of a fiber bundle and less movement perpendicularly. To estimate the nine tensor matrix elements required for a Gaussian description of water mobility, the diffusion gradient must be applied to at least six noncollinear directions. The eigenvalues represent the three principal diffusion coefficients measured along the three coordinate directions of the ellipsoid. The eigenvectors represent the directions of the tensor. Diffusion tensor imaging (DTI) can resolve the dominant fiber orientation in each voxel element. The direction of greatest diffusion measured by DTI parallels the dominant orientation of the tissue structure in each voxel, representing the mean longitudinal direction of axons in white matter tracts. DTI can be applied to identify major white matter tracts, such as the pyramidal tract or the visual pathway $[1,6,17]$.

Avoiding postoperative neurological deficits by preserving eloquent brain areas is a major principle in neurosurgery. Cortical eloquent brain areas can be preserved successfully by identification of these areas by methods such as magnetoencephalography (MEG) and functional magnetic resonance imaging (fMRI). Registration of MEG and fMRI data with 3-D anatomical MR datasets allows representation of the spatial information of the identified cortical eloquent brain areas in the surgical field by using neuronavigation systems [9, 15, 22]. Heads-up displays visualizing segmented data in the surgical field are routinely used in so-called microscope-based neuronavigation. While MEG and fMRI only identify cortical eloquent brain areas, DTI can be used to assess the major white matter tracts connecting to these cortical brain areas $[4,10,11]$. These major white matter tracts have also to be preserved during surgery to avoid postoperative deficits. Different visualization techniques of DTI data, such as glyph representation and fiber tracking have evolved in the recent years. The aim of this 
study is to find a suitable method to visualize the course of major white matter tracts during neurosurgical procedures.

\section{Methods}

Single-shot spin-echo diffusion weighted echo planar imaging on a $1.5 \mathrm{~T}$ MR scanner (Magnetom Sonata, Siemens Medical Solutions, Erlangen, Germany) was used for DTI. The sequence parameters were: TE 86 ms, TR $9200 \mathrm{~ms}$, matrix size $128 \times 128$, FOV $240 \mathrm{~mm}$, slice thickness $1.9 \mathrm{~mm}$, bandwidth $1502 \mathrm{~Hz} / \mathrm{Px}$. A diffusion weighting of $1000 \mathrm{~s} / \mathrm{mm} 2$ (high $\mathrm{b}$ value) was used. One null image (low b value: $0 \mathrm{~s} / \mathrm{mm} 2$ ) and six diffusion weighted images were obtained with the diffusion-encoding gradients directed along the following axes $( \pm 1,1,0),( \pm 1,0,1)$, and $(1, \pm 1,0)$. The voxel size was $1.9 \times 1.9 \times 1.9 \mathrm{~mm}$; 60 slices with no intersection gap were measured. Applying 5 averages the total DTI measurement required 5 minutes and 31 seconds.

Directly after image acquisition, the DTI data could be evaluated with the DTI task card version 1.7 (Magnetic Resonance Center, Massachusetts General Hospital, Boston) on a Siemens scanner using MR software MRease N4_VA21B under syngo VB10I. The diffusion tensor information could then be represented as color-encoded fractional anisotropy (FA) maps, which were generated by mapping the principal eigenvector components into red, green, and blue color channels, weighted by fractional anisotropy. Assuming the patient is lying in supine position and the head is not tilted, then the color mapping defines white matter tracts oriented in an anterior/posterior direction in green, a left/right direction in red, and a superior/inferior direction in blue (Fig. 1).

Furthermore, 3-D fibertracking was generated applying a knowledge-based multiple-ROI (region of interest) approach with user-defined seed regions. Tracking was initiated in both retro- and orthograde directions according to the direction of the principal eigenvector in each voxel of the ROI. These data could be displayed on a screen in the operating theatre. Fiber tracts were visualized as streamtubes along the B0 images in coronal, sagittal or axial orientation (Fig. 2).

In parallel DTI data were displayed using an in-house visualizing platform, allowing generating different glyph and fibertract representations, such as evenly spaced streamlines (Fig. 3). Additionally standard anatomical image data could be 
registered, so that glyph or streamline fiber tract visualization could be displayed along standard anatomical image data (Fig. 4).

To integrate fibertracking in a navigation system (iPlan 2.5, BrainLab, Heimstetten, Germany) the $b=0$ images were rigidly registered with the T1weighted MPRAGE data (magnetization prepared rapid acquisition gradient echo sequence; TE $4.38 \mathrm{~ms}$, TR $2020 \mathrm{~ms}$, matrix size 256 x 256, FOV $250 \mathrm{~mm}$, slice thickness $1 \mathrm{~mm}$, slab $16 \mathrm{~cm}$, measurement time $8 \mathrm{~min} 39 \mathrm{~s})$. For fiber tracking we implemented a tracking algorithm based on a local diffusion approach. The algorithm is based on a tensor deflection algorithm where the trend of the current generated fibers is also considered, which was first described by Lazar, Weinstein, and Westin [16, 28, 29]. In contrast to the syngo approach (see above) tract seeding is performed by defining a rectangular volume of interest (VOI) either in the FA maps or in the co-registered standard anatomical datasets. The definition of the VOI depends on the fiber structures to be displayed. A raster of a third of the voxel size is applied in the VOI to define the starting points. The tract is propagated both in the forward and reverse directions of the major eigenvector, so that each fiber consists of two iterative calculated segments beginning at the starting point. After termination of the iteration process the calculated fibers must meet all general conditions regarding threshold, local angulation, and total fiber length. Before tracking is initiated the user can adjust the FA threshold and the minimum fiber length. The final result of the tracking calculations is a parametric display of fibers, which are represented as streamlines, using the standard direction color encoding: left-right oriented fibers are displayed in red, anteriorposterior in green, and cranio-caudal in blue (Fig. $5 \mathrm{~A} / \mathrm{B}$ ). For further selecting the fiber bundle of interest, it is possible to define a volume of interest in the initially tracked fibers, so that among this VOI fibers can be retained, excluded, and deleted. This selection of fibers of interest can be repeated as often as necessary, allowing an interactive selection of complex volumes of interest to distinguish single fiber bundles, disconnect branching fibers, or to remove entire areas (Fig. 5 $\mathrm{C} / \mathrm{D})$.

For representation in the navigation software the maximum outline of the fiber tracts was segmented automatically (Fig. 6), so that this object wrapping all fibers is displayed as a single object and could be displayed on the navigation screen (Fig. 6 D), as well as in the surgical field. 


\section{Results}

In over 50 patients, most of them undergoing glioma surgery, DTI data were visualized for intraoperative use. Fig. 1- 6 depict typical examples of the different tools and visualization strategies in the same patient. Table 1 summarizes the clinical suitability for the different visualization techniques.

The DTI task card integrated in the MR scanner syngo interface allowed besides standard display of FA maps (Fig. 1) an immediate visualization of the major fiber tracts (Fig. 2), so that this information could be displayed during neurosurgical procedures, even if these data were acquired intraoperatively. However, this visualization lacked the parallel display of high-resolution anatomy, so that the direct 'mental' transfer of the course of the fiber tracts to the surgical field depended much on the clinical experience of the individual surgeon.

FA map representation (Fig. 1) and glyph representation (Fig. 3) of the data was only used to define the seed regions for the tracking algorithm. This visualization method is of value and interest when displaying the tumor border and comparing this information to other modalities such as MR spectroscopy or PET. Furthermore, FA maps are of great value, when DTI data are used to investigate invasion of major white matter tracts by a tumor by analysis of the various DTI parameters.

Fiber tract representation of DTI data was the preferred display mode (Fig. 2). However, the standard display with smoothed streamlines, suggested a high accuracy and resolution of the data, which effectively is not provided by the voxel size of $1.9 \times 1.9 \times 1.9 \mathrm{~mm}$ of the actually measured data. What was most important was the simultaneous display of fiber tract data along with standard anatomical images, allowing a clear spatial orientation (Fig. $4 \& 5$ ).

Integration of DTI information into navigational systems, either by registration of the color-encoded FA maps and manual segmentation of the respective fiber tracts by an expert (applied in 16 glioma patients) or by integrating the reconstructed streamlines in the navigational setup $(n=20)$ allowed a direct visualization of the pyramidal tract or the optic radiation in the surgical field. The fiber tract generation and integration into the navigation system needed about 25-30 minutes in each case. Integrating the reconstructed streamlines in the navigational setup was less time consuming and less user dependent, i.e. less biased than the approach applying the direct registration of color-encoded FA maps. Final 
visualization of a 3-D representing the major white matter tract of interest proved to be most suitable for intraoperative visualization (Fig. 6). For the visualization in the surgical field, using the heads-up display systems of navigation microscopes a smooth wrapping of all fibers of interest proved to be of major importance, otherwise the displayed structures (contours) in the surgical field could not be related easily to the structures of interest.

DTI data navigation allowed extended resections with low new postoperative deficits; only in one patient $(2.8 \%$; 1 of 36$)$ we observed a permanent new postoperative neurological deterioration.

\section{Discussion}

DTI data are of increasing interest for neurosurgeons since DTI provides information about the normal course, the displacement, or interruption of white matter tracts around a tumor, as well as a widening of fiber bundles due to edema or tumor infiltration can be detected $[3,4,10,18,25,30]$. Various visualization approaches have emerged in the recent years, however, up to now there is no common standard how DTI data are presented during neurosurgical procedures. In the following the major different visualization strategies are discussed.

\section{Glyphs}

The simplification of a tensor to a scalar metric reduces the amount of information contained in the tensor. A possibility to show the entire information of the second order tensors is to use glyphs. Several shapes have been presented for representing tensors using glyphs [24]. A popular approach is to use ellipsoids whereas the axes of the ellipsoid correlate with the directions of the eigenvectors and are scaled according to the corresponding eigenvalues. An advantage of tensor ellipsoids is that they can be rendered in real-time using graphics hardware [7]. An even more satisfying shape for tensors are superquadric tensor glyphs which provide a better and less ambiguous spatial impression [14]. However, superquadrics are computationally much more expensive and are hard to achieve in real-time.

The advantage of glyph-based techniques is that the entire tensor information is visualized. Therefore, it is especially appropriate for a detailed examination of the data. However, the results of this visualization approach are difficult to interpret 
in terms of underlying major white matter structures since no global connectivity information is provided. Consequently, a combination with other visualization techniques such as fiber tracing is recommended to obtain a meaningful representation. In addition, clipping or slice views of glyphs are necessary to avoid an overloading and cluttering of information in $3 \mathrm{D}[7,14]$.

\section{Fiber Tracking}

Fiber tracking which is maybe the most appealing and understandable technique for representing white matter has been investigated by several groups $[2,8,20$, 26, 27, 31]. All these approaches are based on streamline techniques known from flow visualization. Thereby, the respective vector field is derived by taking the major eigenvector of each tensor. Fiber tracking algorithms often utilize thresholds, angle criterions, regularization techniques and local filters to improve tracking results.

In standard fiber tracking approaches the streamlines extracted by tracking algorithms are assumed to represent the most likely pathways through the tensor field. Note that the term 'fibers' is used for streamlines which do not represent real anatomical fibers but provide an abstract model of neural structures. Starting from seed voxels, the tracking is performed in forward and backward direction with sub-voxel precision. For the selection of seed voxels and for aborting the streamline propagation, FA is used as a threshold. FA represents the degree of anisotropic diffusion and therefore is a proper measure for the probability of white matter. Following this assumption, voxels with high FA are used as seed voxels. If FA falls below a certain threshold, the tracking stops.

Accordingly, a single tracking step of the streamline propagation looks as follows: The tensor at the current end point of the fiber is computed using trilinear interpolation which is separately performed for each tensor entry. The eigensystem of the tensor is then determined. The eigenvector belonging to the highest eigenvalue, in the following referred to as principal eigenvector, correlates with the direction of highest diffusion. In case of Euler integration, the next streamline propagation step would be in direction of this principal eigenvector. For reasons of numerical accuracy, we apply a higher order integration scheme (Runge-Kutta of order four) which needs repeated tensor interpolation and principal eigenvector computations until the direction of streamline propagation is 
determined. The step size is set to a fixed value which is a quarter of the voxel size. Since the field of the principal eigenvectors does not correspond to a flow field we found it more convenient to choose a sufficiently low fixed step size instead of adaptive adjustments. Contrarily to real fibers, particle flow posses physical properties such as inertia which ensure that sudden changes of direction do not occur. For fiber tracking a fixed step size is safer to prevent missing turnoffs. Apart from the FA threshold for aborting fiber tracking, streamline propagation is aborted in the subsequently described cases. If a streamline has reached a maximum length or if the angle between the last two steps is above a certain threshold, tracking stops. A further criterion for accepting a fiber is its length. Streamlines are rejected if they are below a minimum length. Beside these thresholds, the user may choose between a tracking encompassing the whole brain and a tracking extracting fibers that run through user-defined regions of interest. The latter approach enables the reconstruction of separate tract systems which is of special interest to delineate major white matter tracts.

Meanwhile standard fiber tracking techniques provide a straightforward possibility to visualize major white matter tracts and their spatial relation to intracerebral lesions $[6,13,21]$. However, a lot of software tools allow only tracking combined with the display of the diffusion data; co-registration with standard anatomical data is a must for an intuitive analysis in space-occupying lesions.

\section{Evenly spaced streamlines}

Due to the diverging nature of tract systems, the density of streamlines varies over the domain without control resulting in sparse areas as well as cramped regions. To overcome this problem, the concept of evenly spaced streamlines [12] was adapted to fiber tracking providing streamlines equally distributed over the domain. An adaptive control of the distance between separate streamlines depending on the magnitude of anisotropic diffusion provides a mechanism to emphasize dominant tract systems. The basic principle of evenly spaced streamlines is to calculate streamlines until a user-defined density level is reached. Thereby, a regular distribution of the streamlines is achieved and areas with a sparse distribution of streamlines are filled. In the context of fiber tracking, this is a highly desirable property to capture all features, i.e. the location of white matter, 
of the diffusion tensor field. Up to now, this technique does not provide further advantages compared to standard techniques, looked at from the intraoperative visualization viewpoint. Evenly based streamlines are an interesting tool to further investigate tracking techniques per se and may be interesting to more userindependent extract major white matter tracts.

\section{Streamtubes}

The visualization of streamlines is solved by rendering lines or more significant tubes. However, utilizing simple line rendering has several disadvantages. In case of curved fibers, lines thicker than one pixel suffer from gaps between neighboring segments. Furthermore, a constant width independent of the zoom restricts the quality of visualization. Tubes on the other hand enable a significantly improved visualization, but rendering becomes much more time consuming due to the high geometric complexity restricting their interactive application. Alternatively, streamline visualizations which are very similar to tubes are available providing interactive rendering times since no complex geometry is necessary.

\section{Hulls}

So far, all presented approaches are motivated by the exploration of the diffusion tensor data. Unfortunately, they do not satisfy the needs of neurosurgical planning. In this case, the problem is the exact determination of the border of a specific major white tract. Line representation lacks the ability to provide a border. Instead, it is the job of the user to interpret the visualization as a model for the tract. The generation of hulls is a possibility to overcome this issue [7]. Thereby, a surface is generated which wraps a particular subset of previously computed streamlines, representing a certain nerve tract. The wrapping itself is based on the determination of a centerline of the bundle and the subsequent construction of bounding curves around the set of corresponding lines. Finally, a mesh is generated by connecting the curves, representing the surface of the nerve tract model.

The major advantage of this method is the intuitive visualization. The results look like flexible tubes which are closely related to the expected appearance of a major white matter bundle. The borders are depicted directly and the combination with 
volume rendering of anatomical MR data provides a good spatial orientation. It must be noted that the surface is entirely dependent on the previously performed fiber tracing. Errors in the streamline calculation have immediate influence on the resulting hull.

\section{Volume Growing}

In general, volume growing algorithms start from a predefined seed region and spread out within the volume until some terminating criterion is reached. Similarly to ROI-based fiber tracking, volume growing needs an initial ROI for starting the growing procedure. The algorithm starts from each voxel within the ROI and proceeds to neighboring voxels. In the same way as for fiber tracking, the growing process stops as soon as a FA value below a specified threshold is reached. In addition, directional volume growing takes into account the shape of the local tensor which controls the direction in which the process expands [19].

The advantage of volume growing approaches over isosurface based approaches is that a connected volume is generated. The fragmented nature of an isosurface based on the FA volume makes it difficult to clearly identify connectivity information which is important for surgery. In contrast, volume growing produces a connected volume without any detached parts.

\section{Illumination}

A general problem of all 3-D visualization approaches is the projection into 2-D screen space. To overcome this limitation some further dodges are necessary. Beside the perspective projection, the appliance of light is an important factor to receive a good 3D impression. Thereby, the color of a structure is modified dependent on the orientation with respect to the light source. Further on, specular highlights and shadows significantly contribute to the visual perception of 3 dimensional structures. In general, this is also valid for the case of DTI visualization. Hulls and glyphs highly benefit from the use of illumination since they have a spatial structure and a surface. In particular, detailed surface structures, as they appear on hulls, can hardly be distinguished by the user without the application of light. In contrast to that, the use of light in combination with lines is problematic. Since lines do not have an explicit surface, illumination can only be achieved by the simulation of infinitesimal small tubes. For correlating 
lines this works satisfactorily and the highlights look expectedly. In case of turbulent lines or line bundles which fan out, the results look extremely scattered and the illumination is counterproductive [32].

\section{Clinical application and intraoperative use}

Integration of DTI data into navigation systems allowing an immediate visualization in the surgical field has resulted in resections with low morbidity [5, 13, 23]. We have successfully implemented a fiber tracking algorithm in a common navigation software environment, allowing broad availability and providing a common platform to compare the results of different groups.

Our current study showed that for intraoperative visualization, a single object representing the major white matter tract of interest, such as e.g. the pyramidal tract, proved to be most efficient for a reliable intraoperative orientation. This 3-D object can be displayed along with other objects representing the tumor outline on the navigation screen, as well as it can be superimposed as contour in the surgical field. Direct visualization of fiber tract data as sophisticated 3-D renderings applying illuminated streamtubes may be an impressive view for a layperson, however, superimposition of these detailed fiber tract information, suggesting an accuracy of the data, that in fact is not present, would obscure the view for the surgeon and prevent concentrating on the most critical structures. This is the area where a tumor is reaching the nearest point of a major white matter tract system, because entering major white matter tracts has to be avoided during surgery to prevent postoperative new neurological deficits. This explains why the representation as a 3-D object is sufficient for intraoperative use. A promising approach for an even more sophisticated visualization is adding layers to the hulls of the 3-D object with a thickness defined by safety margins. These additional hulls would allow visualizing the quality of the data, as well as representing safety corridors. So a thick hull in case of noisy unreliable data, or a thin hull in the area of high reliability would be added. This would allow that the neurosurgeon has an immediate impression to what extent the displayed data can be trusted during surgery. The technical, as well as clinical definition of the extent of these safety margins has yet to be established.

When integrating DTI data in a neuronavigational setup brain shift has to be compensated for. Intraoperative fiber tracking revealed a marked shifting of major 
white matter tracts during tumor resection [21]. These intraoperative DTI data can be visualized during surgery with the techniques described above; the integration of fiber tracking in standard neuronavigation system makes it even feasible that fiber tract data are update during surgery.

The ideal platform would combine the different features: allowing basic display and analysis of FA maps, including numeric evaluation of the different diffusion parameters, which may be of special interest when looking at the relation of a major white matter tract system and a tumor in respect to edema and tumor invasion; up to integration and co-registration of data from various other modalities, allowing generation of streamtubes and 3-D glyph representations, as well as features defining hulls wrapping major white matter tracts of interest. Furthermore, automatic clustering techniques, facilitating the identification of the white matter tract of interest, should be established. All these data should be integrated in a navigational setup environment, so that the different information can be displayed during surgery in adapted detail and representation. The visualization strategy has to avoid disturbing the neurosurgeon form the surgical field, i.e. preventing an information overflow; however complex data have to be presented in a hierarchical fashion, so that they can be easily used during surgery.

\section{Conclusion}

Streamline representation of major fiber tract systems along with high-resolution anatomical data provides a reliable orientation for the neurosurgeon. For direct visualization of these data in the surgical field applying heads-up displays of operating microscopes, wrapping of all streamlines of interest to render an individual object representing the whole fiber bundle is mandatory. Integration of fiber tract data into a neuronavigation setup allows removal of tumors adjacent to eloquent brain areas with low morbidity. 


\section{Acknowledgements}

We express our special thanks to Greg Sorensen (Dept. Radiology, MGH, Boston, USA) for providing the DTI syngo taskcard software, as well as to G. Ogrezeanu, U. Mezger, and T. Seiler (BrainLab, Heimstetten, Germany) for implementation of the fiber tracking software in the navigation environment; Theodor Vetter, Ph.D., and Michael Zwanger, Ph.D. (Siemens Medical Solutions, Erlangen, Germany) for their continuous technical advice, as well as to Stefanie Kreckel, RT, for her technical support in MR imaging. This work was supported in part by the Deutsche Forschungsgemeinschaft in the context of project C9 of SFB603.

\section{References}

1. Basser PJ, Mattiello J, LeBihan D (1994) MR diffusion tensor spectroscopy and imaging. Biophys J 66: 259-267

2. Basser PJ, Pajevic S, Pierpaoli C, Duda J, Aldroubi A (2000) In vivo fiber tractography using DT-MRI data. Magn Reson Med 44: 625-632

3. Berman JI, Berger MS, Mukherjee P, Henry RG (2004) Diffusion-tensor imaging-guided tracking of fibers of the pyramidal tract combined with intraoperative cortical stimulation mapping in patients with gliomas. J Neurosurg 101: 66-72

4. Clark CA, Barrick TR, Murphy MM, Bell BA (2003) White matter fiber tracking in patients with space-occupying lesions of the brain: a new technique for neurosurgical planning? Neuroimage 20: 1601-1608

5. Coenen VA, Krings T, Mayfrank L, Polin RS, Reinges MH, Thron A, Gilsbach JM (2001) Three-dimensional visualization of the pyramidal tract in a neuronavigation system during brain tumor surgery: first experiences and technical note. Neurosurgery 49 : 86-93

6. Cruz Junior LC, Sorensen AG (2005) Diffusion tensor magnetic resonance imaging of brain tumors. Neurosurg Clin N Am 16: 115-134

7. Enders F, Iserhardt-Bauer S, Hastreiter P, Nimsky C, Ertl T (2005) Hardware-accelerated glyph based visualization of major white matter tracts for analysis of brain tumors. In: Galloway RJ, Cleary K(eds.) Medical Imaging 2005: Visualization, Image-Guided Procedures, and Display. Proceedings of SPIE Vol. 5744, Bellingham, WA, pp 504-511

8. Fillard P, Gilmore J, Lin W, Gerig G (2003) Quantitative Analysis of White Matter Fiber Properties along Geodesic Paths. In: Ellis RE, Peters TM(eds.) Medical Image Computing and Computer-Assisted Intervention - MICCAI 2003. Springer, pp 16-23

9. Ganslandt O, Fahlbusch R, Nimsky C, Kober H, Möller M, Steinmeier R, Romstöck J, Vieth J (1999) Functional neuronavigation with magnetoencephalography: outcome in 50 patients with lesions around the motor cortex. J Neurosurg 91: 73-79 
10. Hendler T, Pianka P, Sigal M, Kafri M, Ben-Bashat D, Constantini S, Graif M, Fried I, Assaf Y (2003) Delineating gray and white matter involvement in brain lesions: threedimensional alignment of functional magnetic resonance and diffusion-tensor imaging. $\mathrm{J}$ Neurosurg 99: 1018-1027

11. Holodny AI, Schwartz TH, Ollenschleger M, Liu WC, Schulder M (2001) Tumor involvement of the corticospinal tract: diffusion magnetic resonance tractography with intraoperative correlation. J Neurosurg 95: 1082

12. Jobard B, Lefer W (1997) Creating evenlyspaced streamlines of arbitrary density. (eds.) Visualization in Scientific Computing '97 Proceedings of the Eurographics Workshop in Boulogne-sur-Mer, France. Springer Verlag, Wien, New York, pp 43-56

13. Kamada K, Todo T, Masutani Y, Aoki S, Ino K, Takano T, Kirino T, Kawahara N, Morita A (2005) Combined use of tractography-integrated functional neuronavigation and direct fiber stimulation. J Neurosurg 102: 664-672

14. Kindlmann G (2004) Superquadric Tensor Glyphs. (eds.) Proc Eurographics - IEEE TCVG Symposium on Visualization. pp 147-154

15. Kober H, Nimsky C, Möller M, Hastreiter P, Fahlbusch R, Ganslandt O (2001) Correlation of sensorimotor activation with functional magnetic resonance imaging and magnetoencephalography in presurgical functional imaging: a spatial analysis. Neuroimage 14: 1214-1228

16. Lazar M, Weinstein DM, Tsuruda JS, Hasan KM, Arfanakis K, Meyerand ME, Badie B, Rowley HA, Haughton V, Field A, Alexander AL (2003) White matter tractography using diffusion tensor deflection. Hum Brain Mapp 18: 306-321

17. Le Bihan D, Van Zijl P (2002) From the diffusion coefficient to the diffusion tensor. NMR Biomed 15: 431-434

18. Lu S, Ahn D, Johnson G, Cha S (2003) Peritumoral diffusion tensor imaging of highgrade gliomas and metastatic brain tumors. AJNR Am J Neuroradiol 24: 937-941

19. Merhof D, Hastreiter P, Nimsky C, Fahlbusch R, Greiner G (2005) Directional volume growing for the extraction of white matter tracts from diffusion tensor data. In: Galloway RJ, Cleary K(eds.) Medical Imaging 2005: Visualization, Image-Guided Procedures, and Display. Proceedings of SPIE Vol. 5744, Bellingham, WA, USA, pp 165-172

20. Mori S, Van Zijl PC (2002) Fiber tracking: principles and strategies - a technical review. NMR Biomed 15: 468-480

21. Nimsky C, Ganslandt O, Hastreiter P, Wang R, Benner T, Sorensen AG, Fahlbusch R (2005) Preoperative and intraoperative diffusion tensor imaging-based fiber tracking in glioma surgery. Neurosurgery 56: 130-138

22. Nimsky C, Ganslandt O, Kober H, Möller M, Ulmer S, Tomandl B, Fahlbusch R (1999) Integration of functional magnetic resonance imaging supported by magnetoencephalography in functional neuronavigation. Neurosurgery 44: 1249-1256

23. Nimsky C, Grummich P, Sorensen AG, Fahlbusch R, Ganslandt O (2005) Visualization of the pyramidal tract in glioma surgery by integrating diffusion tensor imaging in functional neuronavigation. Zentralbl Neurochir 66: 133-141 
24. Pierpaoli C, Jezzard P, Basser PJ, Barnett A, Di Chiro G (1996) Diffusion tensor MR imaging of the human brain. Radiology 201: 637-648

25. Price S, Burnet N, Donovan T, Green H, Pena A, Antoun N, Pickard J, Carpenter T, Gillard J (2003) Diffusion tensor imaging of brain tumours at 3T: a potential tool for assessing white matter tract invasion? Clin Radiol 58: 455-462

26. Stieltjes B, Kaufmann WE, van Zijl PC, Fredericksen K, Pearlson GD, Solaiyappan M, Mori S (2001) Diffusion tensor imaging and axonal tracking in the human brainstem. Neuroimage 14: 723-735

27. Vilanova A, Berenschot G, Pul van C (2004) DTI Visualization with Streamsurfaces and Evenly-Spaced Volume Seeding. (eds.) Proc Joint EG/IEEE TCVG VisSym. pp 173-182

28. Weinstein DM, Kindlman GL, Lundberg EC (1999) Tensorlines: advection-diffusion based propagation through diffusion tensor fields. In: Ebert DS, Gross MH, Hamann B(eds.) IEEE Visualization 1999. IEEE Computer Society and ACM, San Francisco, pp 249-253

29. Westin CF, Maier SE, Mamata H, Nabavi A, Jolesz FA, Kikinis R (2002) Processing and visualization for diffusion tensor MRI. Med Image Anal 6: 93-108

30. Yamada K, Kizu O, Mori S, Ito H, Nakamura H, Yuen S, Kubota T, Tanaka O, Akada W, Sasajima H, Mineura K, Nishimura T (2003) Brain Fiber Tracking with Clinically Feasible Diffusion-Tensor MR Imaging: Initial Experience. Radiology 227: 295-301

31. Zhukov L, Barr A (2002) Oriented Tensor Reconstruction: Tracing Neural Pathways from Diffusion Tensor MRI. (eds.) VISUALIZATION Proceedings of the conference on Visualization '02. IEEE Computer Society, Washington, DC, USA, pp 387 - 394

32. Zöckler M, Stalling D, Hege H-C (1996) Interactive visualization of 3D-vector fields using illuminated stream lines. (eds.) IEEE Visualization - Proceedings of the 7th conference on Visualization '96. IEEE Computer Society Press, Los Alamitos, CA, USA, pp 107-113 


\section{Tables}

Table 1 different visualization techniques of DTI data

\begin{tabular}{|l|c|c|c|c|}
\hline & $\begin{array}{c}\text { detailed } \\
\text { analysis }\end{array}$ & $\begin{array}{c}\text { spatial } \\
\text { analysis }\end{array}$ & $\begin{array}{c}\text { preoperative } \\
\text { planning }\end{array}$ & $\begin{array}{c}\text { intraoperative } \\
\text { visualization }\end{array}$ \\
\hline $\begin{array}{l}\text { fractional anisotropy } \\
\text { maps }\end{array}$ & + & - & $\mathrm{O}$ & - \\
\hline glyphs & + & $\mathrm{O}$ & - & - \\
\hline volume growing & $\mathrm{O}$ & + & + & + \\
\hline fiber tracking & - & + & + & - \\
\hline $\begin{array}{l}\text { evenly spaced } \\
\text { streamlines }\end{array}$ & - & + & + & ++ \\
\hline hulls & - & + & + & ++ \\
\hline 3-D objects & - & + & + & + \\
\hline
\end{tabular}




\section{Figures}

Fig. 1 DTI visualization on a 28-years old female patient with a lesion (white arrow) close to the left pyramidal tract, that was biopsied. A: $b=0$ image; B: FA map; C: color-encoded FA map; D: glyph representation of the same FA map by ellipsoids; E: glyph representation of the same slice as quadrics; F: enlarged view of $\mathrm{E}$

Fig. 2 Fiber tract visualization in the same patient applying a streamtube representation displayed along the $\mathrm{b}=0$ images in standard projection (A: coronal, B: sagittal, C: axial).

Fig. 3 Whole head DTI representation, the lesion is segmented in red; A: glyph visualization; BD: streamline visualization, applying different techniques: B: standard tracking with filtering; C: without filtering; D: applying evenly spaced streamlines.

Fig. 4 Simultaneous display of DTI data and standard image data, glyphs or streamlines are displayed along a 3-D rendering of a T1-weighted 3-D dataset (A: glyphs; B: streamlines along the lesion segmented in red).

Fig. 5 Implementation of fiber tracking in the navigation software; A: initial tracking starting from seed points in a large VOI covering the left hemisphere; B: enlarged view of the streamline representation of $\mathrm{A} ; \mathrm{C}$ : after excluding some fibers, the fibers belonging to the left pyramidal tract are visualized; D: enlarged view of the streamline representation of $\mathrm{C}$ (note: the axial slice are visualized viewed from above and the coronal slice is visualized viewed from behind).

Fig. 6 Generation of a 3-D object representing the left pyramidal tract; A: final tracking result with streamline representation; B: automatic computation of a hull wrapping all fibers simultaneous display; C: display of the 3-D object without any streamlines; D. actual intraoperative screenshot from the navigation screen depicting the 3-D object visualization during surgery (note: the axial slice are visualized viewed from above and the coronal slice is visualized viewed from behind). 

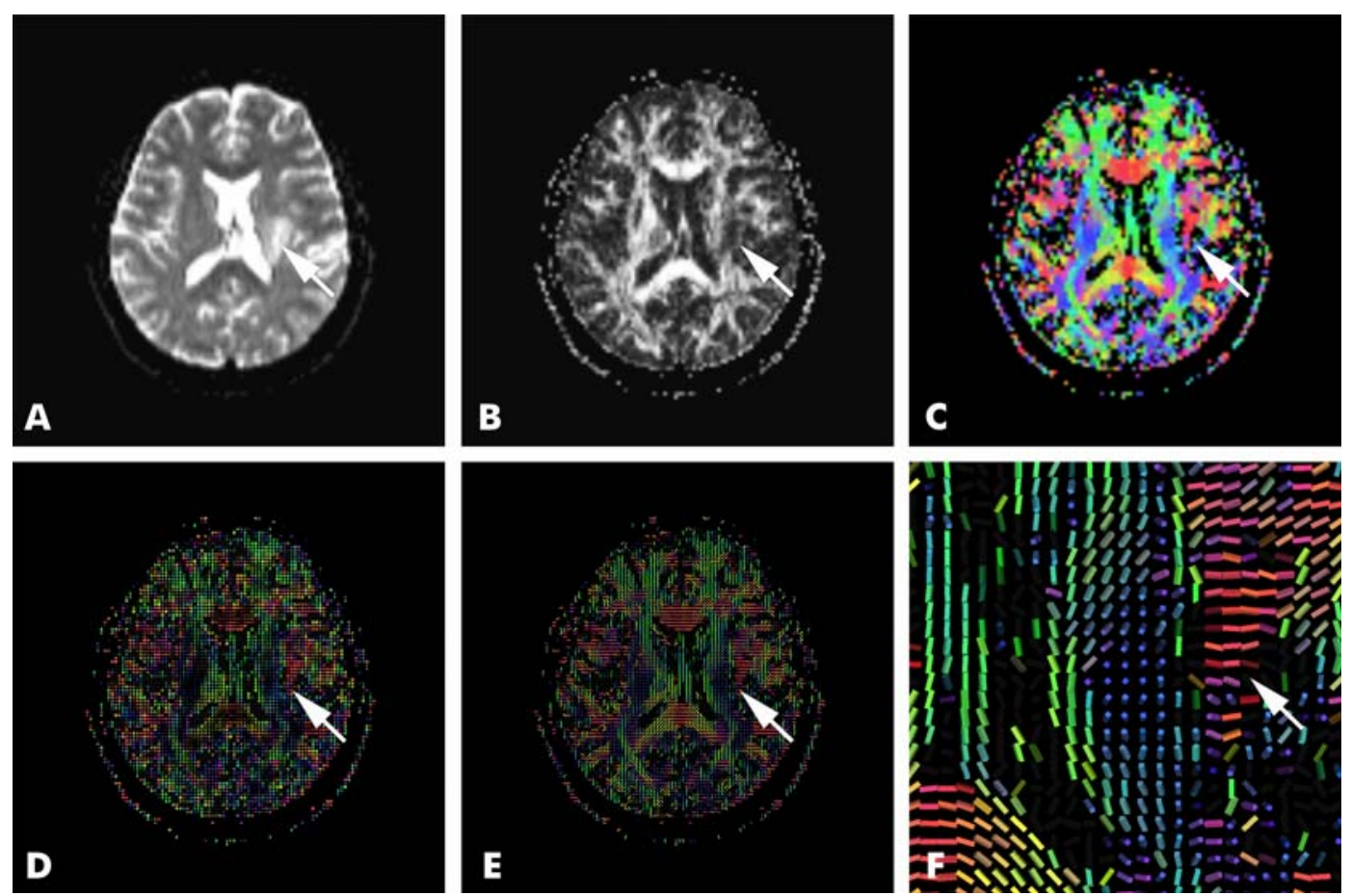

Fig. 1
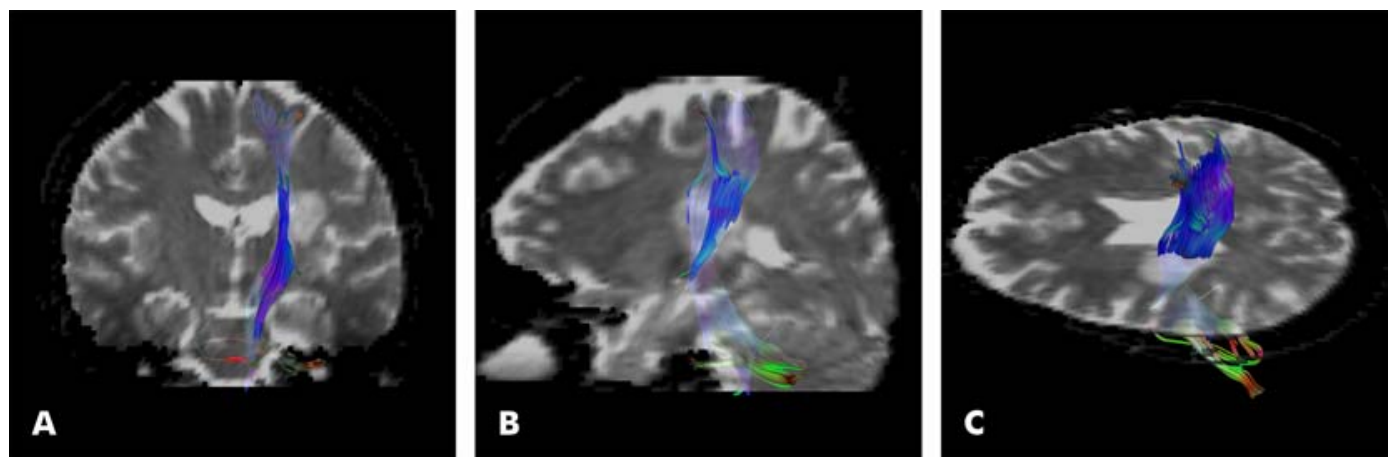

Fig. 2 

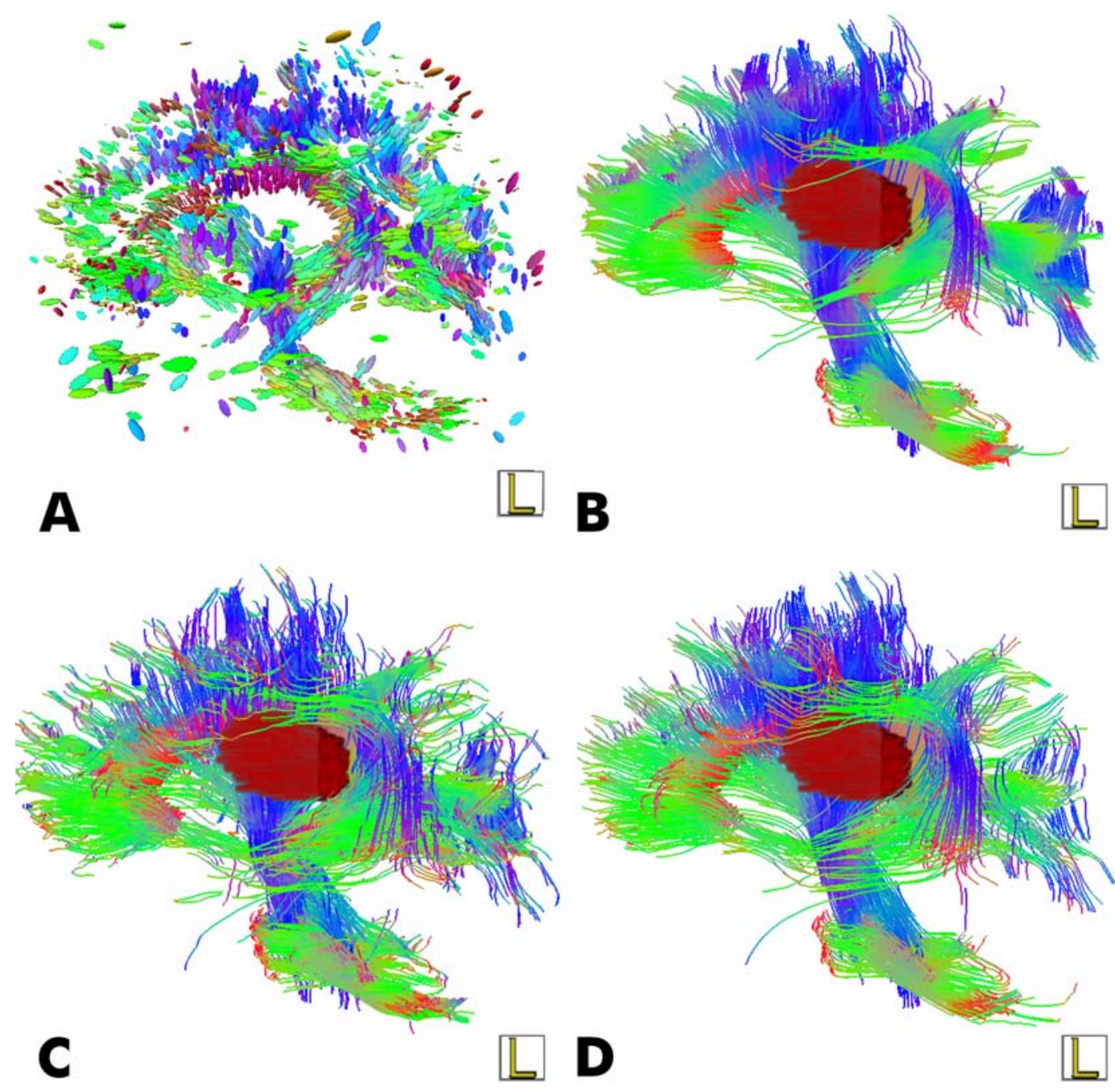

Fig. 3
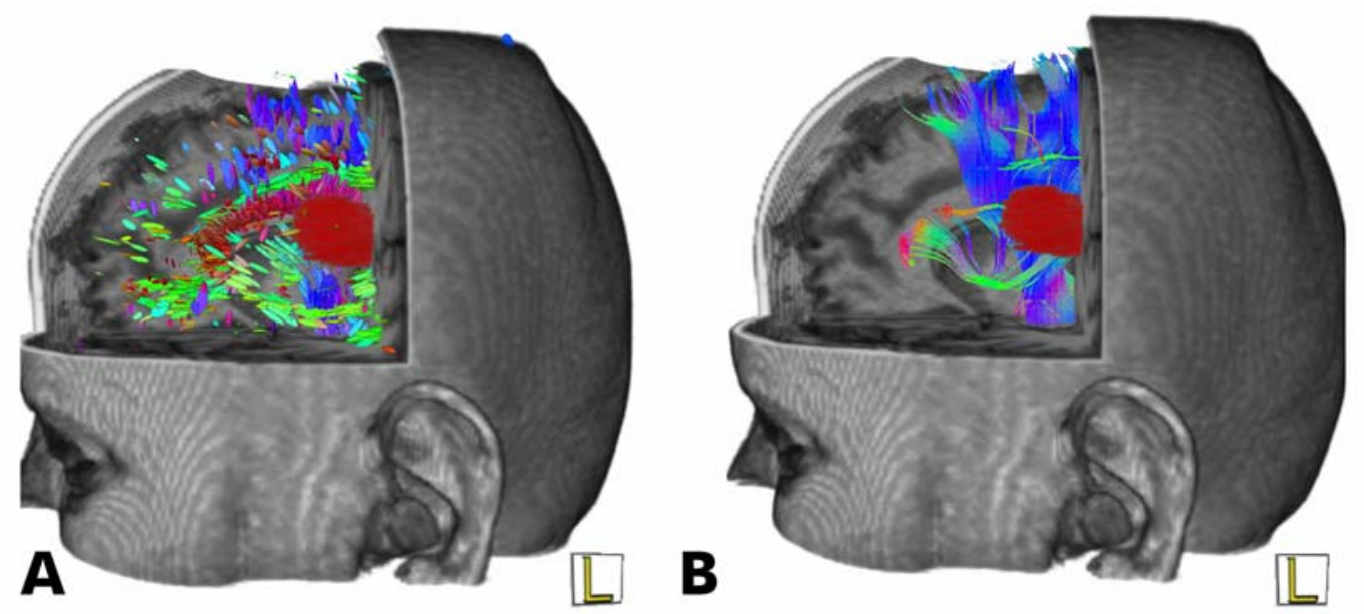

Fig. 4 

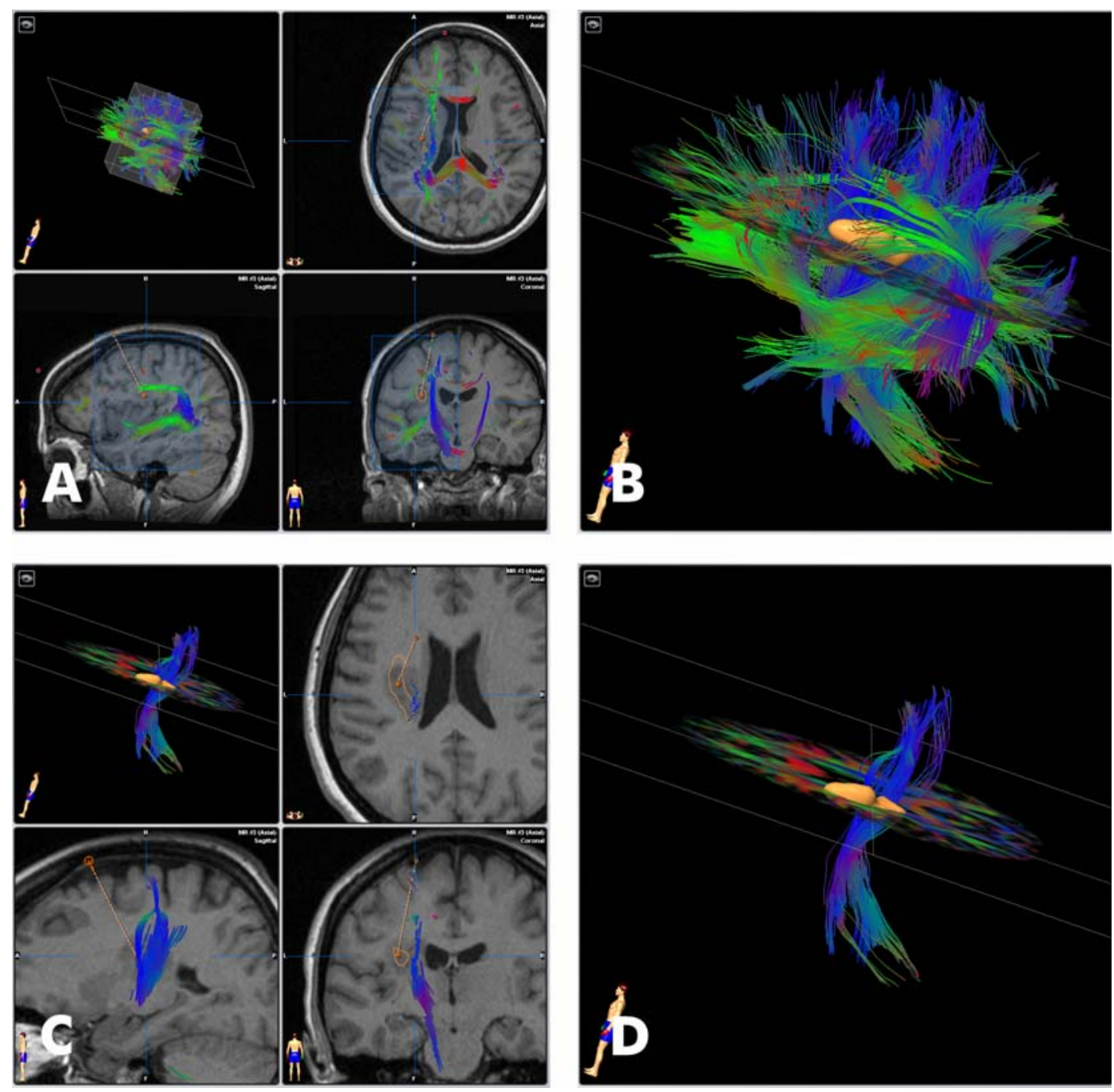

Fig. 5 

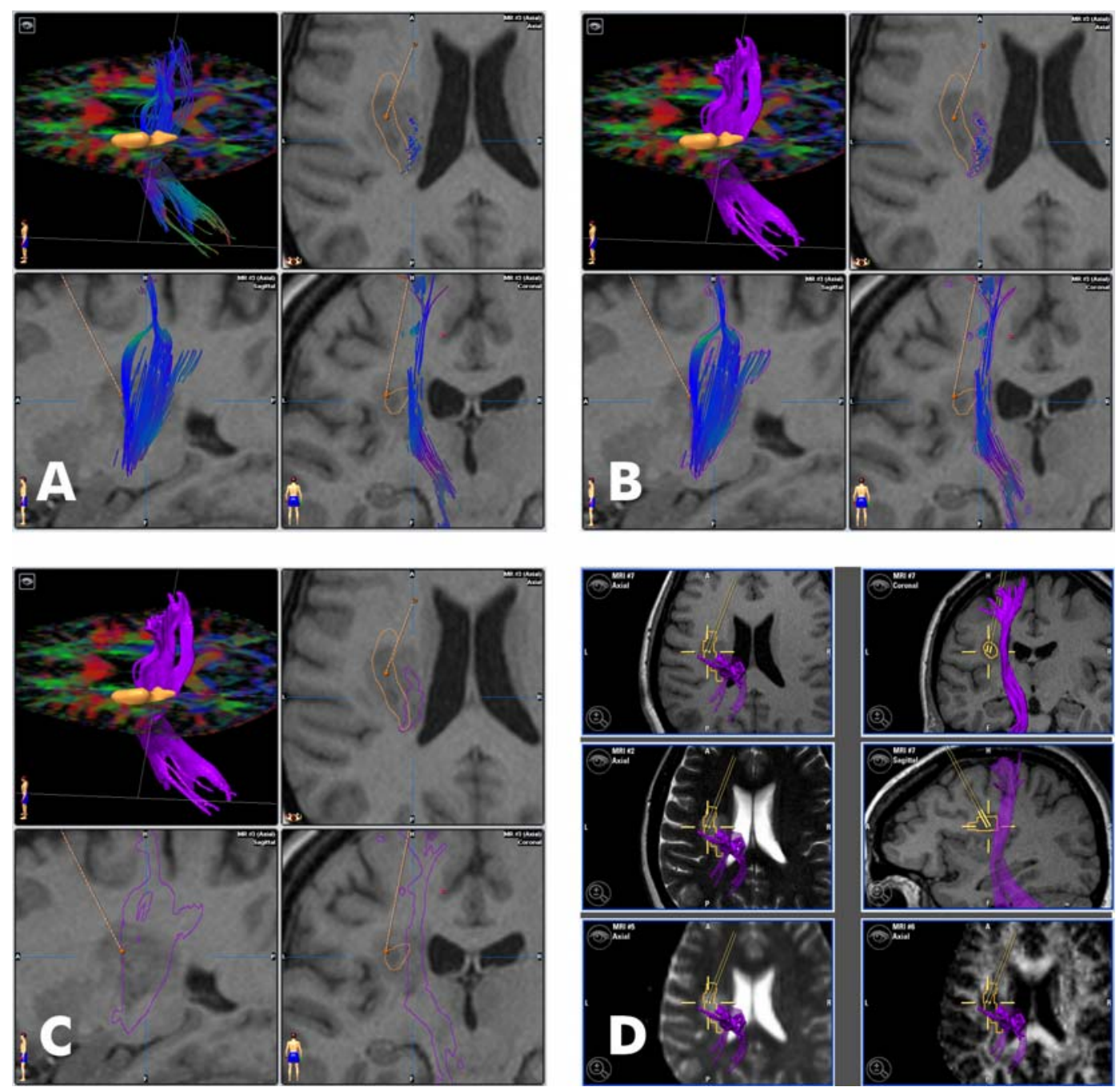

Fig. 6 